\title{
Pyruvate Kinase PKM Isoform M2 Dimer
}

National Cancer Institute

\section{Source}

National Cancer Institute. Pyruvate Kinase PKM Isoform M2 Dimer. NCI Thesaurus.

Code C136626.

A homodimer of the pyruvate kinase PKM isoform M2. This enzyme complex promotes cellular metabolism and is overexpressed in tumors. 